\title{
La apropiación de la enseñanza y el aprendizaje de futuros docentes durante el curso de Didáctica de la Física
}

\author{
Laura Buteler iD \\ Instituto de Física Enrique Gaviola y Facultad de Matemática, Astronomia, Física y Computación, \\ Universidad Nacional de Córdoba.Córdoba.Argentina.laura.butelen@unc.edu.ar \\ Carolina Nieva iD \\ Facultad de Matemática, Astronomia, Física y Computación. Universidad Nacional de Córdoba. Córdoba. \\ Argentina.nievamo@gmail.com \\ Juan Velasco iD \\ Instituto de Investigaciones en Físico-Química de Córdoba y Facultad de Matemática, Astronomía, Física y \\ Computación. Universidad Nacional de Córdoba.Córdoba.Argentina.juan.velasco@unc.edu.ar
}

[Recibido: 27 febrero 2021. Revisado: 14 abril 2021. Aceptado: 30 abril 2021]

Resumen: El profesorado de ciencias se encuentra ante un enorme desafío al momento de re-significar sus concepciones de enseñanza y de aprendizaje durante su formación inicial. Este desafío se genera a partir de las profundas diferencias que existen entre las experiencias previas de los futuros profesores como estudiantes a lo largo de su vida y las concepciones actuales sobre la enseñanza y el aprendizaje provenientes de la didáctica de las ciencias. Este trabajo reporta la apropiación lograda por estudiantes del profesorado de física en relación a las concepciones de enseñanza y de aprendizaje, a lo largo del curso de Didáctica de la Física. La motivación de reportar estos resultados surgió al advertir que, luego de una reformulación metodológica de la asignatura por motivos ajenos a esta investigación, los estudiantes se habían involucrado en ella de manera auténtica, tanto a nivel personal como a nivel colectivo, de un modo inesperado para los docentes del curso. Inspirados en el concepto de apropiación proveniente de la literatura sobre el aprendizaje desde una visión sociocultural, se adoptó una definición conveniente para interpretar el fenómeno ocurrido. Los resultados muestran que la definición de apropiación utilizada se adaptó muy bien a los registros disponibles, permitiendo reconocer apropiación en cinco de los seis futuros docentes involucrados, y que las ideas auténticas e idiosincráticas que definen el sello personal de cada estudiante coinciden con concepciones contemporáneas sobre aprendizaje y enseñanza provenientes de la didáctica de las ciencias.

Palabras clave: formación docente inicial; apropiación; aprendizaje; enseñanza; didáctica de la física

The appropriation of teaching and learning achieved by pre-service teachers in a Didactics of Physics course

Abstract: Science teacher training programs face a big challenge when it comes to re-signifying trainees' ideas of what it means to teach and to learn. This challenge is generated by the profound differences that exist between prospective teachers' previous experiences as lifelong learners and the current conceptions of teaching and learning coming from science education. This paper reports the appropriation of such conceptions achieved by pre-service physics teachers, throughout the course Didactics of Physics, which is part of their training. The motivation to report these results was the observation that, after a methodological reformulation of the course proposed for purposes unrelated to this research, students showed a deeper commitment to those conceptions, both at a personal and a collective level, in ways that went beyond the expectations of the course' instructors. Inspired by the concept of appropriation, from the literature on sociocultural learning, the authors adapted a suitable definition to interpret the phenomenon that occurred during the course. Results showed that the definition of appropriation used fit very well to the students' academic assessments and reflections, and allowed us to recognize appropriation in five of the six future teachers. Also, that the authentic and idiosyncratic ideas that define each pre-service teacher coincided with contemporary conceptions of learning and teaching from science education.

Keywords: pre-service teachers' development; appropriation; leaning; teaching; didactics of physics

Para citar este artículo: Buteler L., Nieva C. y Velasco J. (2021) Apropiación de la enseñanza y el aprendizaje de futuros profesores de física durante el curso de Didáctica de la Física. Revista Eureka sobre Enseñanza y Divulgación de las Ciencias 18(3), 3601. doi: 10.25267/Rev_Eureka_ensen_divulg_cienc.2021.v18.i3.3601 


\section{Introducción}

Los futuros docentes llegan a los cursos del profesorado con ideas previas (muchas de ellas implícitas) en relación a qué es enseñar y qué es aprender. Estas ideas se conciben intuitivamente a partir de las experiencias de los futuros docentes durante sus trayectorias escolares previas. En Argentina, el primer momento de cuestionamiento y tensión entre esas ideas y las sostenidas por las corrientes didácticas contemporáneas, ocurre en los espacios curriculares de las didácticas específicas. En este sentido, podríamos decir que la semilla (de una parte) del Conocimiento Didáctico del Contenido (CDC) comienza a desarrollarse en los espacios destinados a las didácticas específicas (Melo Niño et al. 2016, Mellado et al. 2014, Magnusson et al. 1999). El CDC es más complejo que el conocimiento sobre las orientaciones para la enseñanza y el aprendizaje de los estudiantes, y requiere del desarrollo y la interrelación de otros conocimientos como el campo disciplinar, los documentos curriculares, las herramientas para la enseñanza, y la dinámica de la comunicación en el aula, entre otros (Acevedo 2009). Sin embargo, arrojar luz sobre cómo son los primeros pasos, o algunos aspectos de esos primeros pasos, del desarrollo del CDC del profesor en formación es un aporte enriquecedor para poder entender el proceso de desarrollo de este conocimiento a mayor escala.

Este estudio intenta hacer un aporte en esa dirección, reportando sorprendentes e inesperados cambios (desde el punto de vista de los docentes del curso) de las concepciones de aprendizaje y de enseñanza de futuros profesores luego de su paso por Didáctica de la Física. Los cambios resultaron sorprendentes porque fueron más allá de los aprendizajes esperados por los docentes del curso, luego de reformular la metodología de trabajo en la asignatura con el propósito de resolver un problema puntual de la formación de los docentes. Si bien este problema puntual se revelaba en el espacio de las prácticas de la enseñanza (al año siguiente de Didáctica de la Física) tenía que ver con un contenido de Didáctica de la Física: las ideas previas de los estudiantes y su relación con el aprendizaje.

En el espacio curricular de la práctica de la enseñanza en el último año de la carrera, los estudiantes de profesorado enfrentaban dificultades para diseñar actividades que tuvieran en cuenta las ideas previas de los estudiantes secundarios. Concretamente, los estudiantes de profesorado tenían en cuenta esas ideas previas sólo al comenzar el desarrollo de los contenidos, recabándolas mediante algún cuestionario o problema planteado para tal fin, pero ignorando el resultado de esta actividad en todas las actividades subsecuentes. Los docentes del espacio de práctica advertían que, aunque el contenido "ideas previas" había sido desarrollado en Didáctica de la Física el año anterior, estos futuros docentes no se habían apropiado del rol fundamental que esas ideas previas juegan en el proceso de aprendizaje de los contenidos de física. Operaba allí como un principio contrario a los enfoques constructivistas para la enseñanza, tan difundidos y enseñados desde hace más de 30 años (Carretero 1997).

Ese fue el motivo por el cual se realizó una innovación metodológica en el curso de Didáctica de la Física. Esta innovación, pensada únicamente con fines docentes para intentar mejorar un aspecto puntual de la enseñanza, tuvo un impacto que fue más allá de promover la comprensión de los futuros docentes sobre el rol de las ideas previas en el proceso de aprendizaje. Los estudiantes se involucraron con la asignatura de una forma especial, desde lo personal, pero también en relación al trabajo colectivo del grupo clase, construyendo una narrativa de sí mismos (identidad) a través de la asignatura. Por esta razón se caracterizó como sorprendentes a los resultados obtenidos.

Existen antecedentes sobre las relaciones entre aprendizaje y construcción de identidad en estudiantes de ciencias de la escuela secundaria. Muchos de ellos han elegido el término apropiación para referirse al nexo entre aprender ciencia y construir identidad (Levrini et al. 2015, Brown 2006, Polman 2006, Tabak y Baumgartner, 2004). En particular Levrini et al. (2015) desarrollaron una definición operacional de apropiación, que les permitió determinar si 
hubo (o no) apropiación de conceptos termodinámicos en estudiantes secundarios. Aquí se adapta esa definición operacional (indicadores) de apropiación, desarrollada en el contexto de estudiantes secundarios aprendiendo física, al contexto de estudiantes de profesorado de física durante el cursado de Didáctica de la Física.

En el siguiente apartado se describe brevemente el cambio de metodología introducido en el curso de Didáctica de la Física realizado con fines docentes para mejorar un aspecto de la enseñanza y que, ante los sorprendentes aprendizajes observados, dio lugar a un proceso investigativo posterior. Luego, a modo de lente teórico para abordar el proceso investigativo, se presenta el concepto de apropiación y su definición operacional (indicadores), elaborada por Levrini et al. (2015). En lo que sigue, se describen las características de los estudiantes del curso involucrados en este estudio y se explicita cuáles de las producciones generadas en el marco del curso de Didáctica de la Física se seleccionaron como registros para el proceso de investigación que se reporta aquí. A continuación, se presenta el proceso metodológico de adaptación y aplicación de los indicadores elaborados por Levrini et al. (2015) a nuestros registros. Seguidamente se presentan los principales resultados obtenidos a través de la lente teórica adoptada. Por último, se presentan algunas conclusiones e implicaciones para la instrucción.

\section{La propuesta metodológica en la asignatura Didáctica de la Física}

En el espacio curricular denominado Didáctica de la Física se trabajan los siguientes contenidos: Las ideas previas y su relación con el aprendizaje, el trabajo experimental en física y en enseñanza de la física, el uso de la historia de la física en la enseñanza, el discurso en el aula y su relación con el aprendizaje, las tecnologías de la información y la comunicación, la observación de la enseñanza y la evaluación. Al finalizar la materia, y como parte del examen final, los estudiantes deben elaborar una unidad didáctica sobre un contenido de física, utilizando lo aprendido durante el curso.

El cambio propuesto no fue sobre los contenidos a trabajar sino sobre la forma de trabajarlos, es decir, fue un cambio metodológico. En términos generales, la metodología de enseñanza de todos los bloques, siguió el esquema que se muestra en la Figura 1. La dinámica de cada bloque comienza con un problema (situaciones problemáticas, preguntas, testimonios de clase), sigue con la socialización de las respuestas de los estudiantes, esas respuestas son tensionadas (por los otros estudiantes y/o por los docentes), luego el docente aporta nuevo conocimiento, después se propone a los estudiantes resolver problemas integradores con aporte de los nuevos conocimientos, y finalmente se los invita a comunicar los resultados.

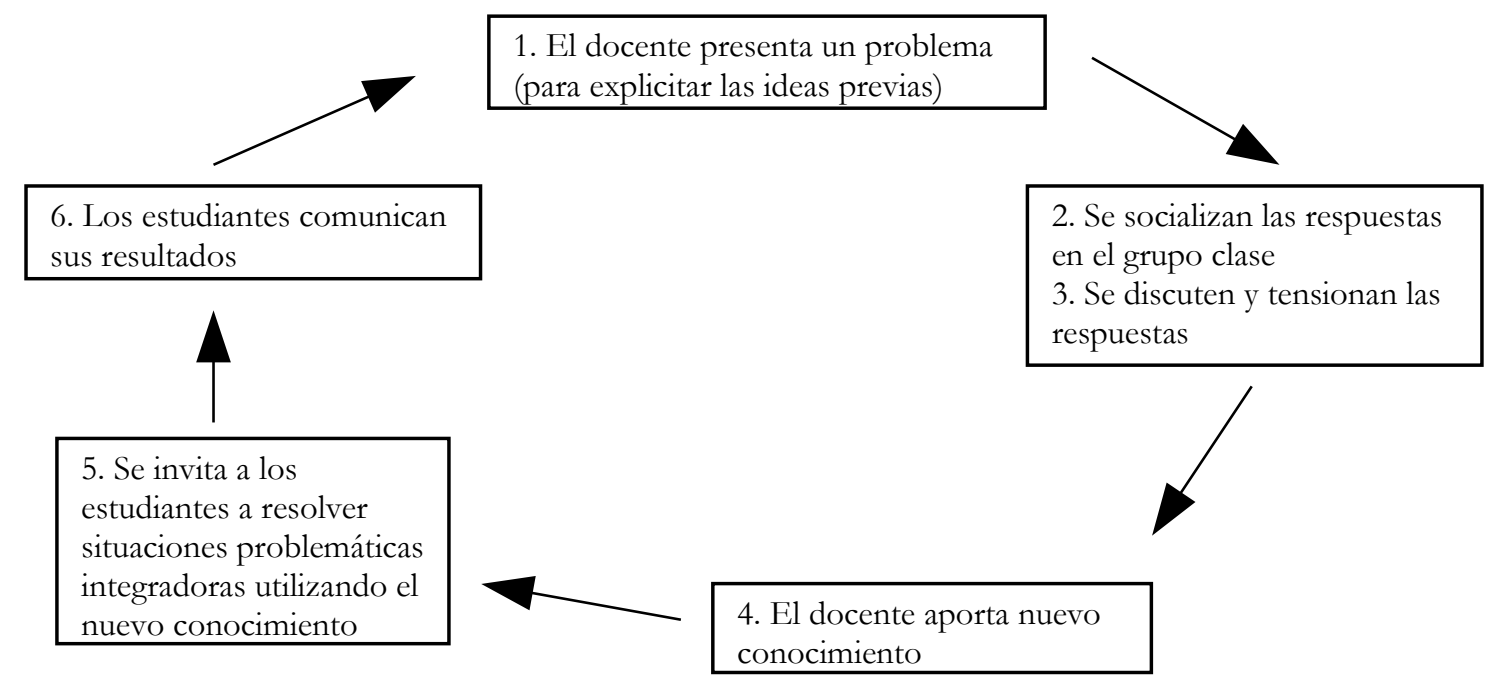

Figura 1. Esquema general de la metodología de enseñanza adoptada. 
Como se expresó anteriormente, esta dinámica de trabajo comienza siempre recabando las ideas previas de los estudiantes en relación a cada uno de los contenidos de la asignatura a partir de situaciones problemáticas o testimonios. Alrededor de estas ideas se organizan los restantes estadios tal como lo muestra la Figura 1. Se prepararon varias actividades para cada uno de los estadios anteriores a trabajar en cada uno de los bloques de la materia de manera que la dinámica de las clases estaba centrada en las producciones de los estudiantes alrededor de esas actividades y en las discusiones en torno a ellas. Los estudiantes trabajaban en alguna de estas tres instancias: lecturas y actividades individuales fuera del horario de clase, trabajo en pequeños grupos en el horario de clase y discusiones, y puestas en común con el grupo clase completo. Las exposiciones del docente eran escasas y ocurrían hacia el final de una clase en la que ya había habido suficiente discusión (generada a partir de las actividades dadas a los estudiantes) sobre el contenido en cuestión. Cada uno de los bloques de contenidos de la asignatura se evaluaba al finalizar su desarrollo, utilizando distintos formatos: evaluaciones individuales escritas, evaluaciones con exposiciones grupales, evaluaciones a partir de entrega de alguna tarea específica, evaluaciones a partir de foros de discusión, etc.

El cambio metodológico fue propuesto para que los estudiantes vivenciaran una enseñanza que tuviera en cuenta sus ideas previas sobre cada temática a trabajar. Se priorizó la coherencia entre la dinámica de las clases de Didáctica de la Física y las clases que se les pedía a los estudiantes que planifiquen al desarrollar la unidad didáctica al final del curso (y eventualmente las clases que desarrollarían durante sus prácticas el año siguiente). En Nieva et al. (2019) se muestra una descripción más detallada de las actividades realizadas y también algunos resultados preliminares de esta innovación.

Como se adelantó en la introducción, la forma en la que los estudiantes se involucraron con la propuesta fue sorprendente, porque fue más allá de los objetivos planteados para la asignatura. Lo hicieron desde una perspectiva personal, pero también en relación al trabajo colectivo del grupo clase, construyendo una narrativa de sí mismos a través del aprendizaje de la materia. Por esta razón, una vez finalizado el curso se inició un proceso investigativo que es el que se reporta aquí. El lente teórico que permitió interpretar lo ocurrido es el concepto de apropiación, entendido como una extensión de la noción de aprendizaje a la construcción de la identidad. En el siguiente apartado se presentan algunas ideas que sirvieron para interpretar lo ocurrido.

\section{La apropiación como una visión sociocultural del aprendizaje}

El término apropiación fue introducido por Bakhtin (1983) en el ámbito de la lingüística para describir el proceso por el cual una persona puebla de significados propios a una dada palabra. Para él, la apropiación es el proceso por el cual una persona adapta una palabra que existe en el habla de los otros a su propia habla, llenándola de intenciones idiosincráticas, improntas y propósitos personales. Desde la perspectiva de Bakhtin, los objetos de apropiación son palabras y oraciones, y puede extenderse, como se mostrará más adelante, a palabras de contenido didáctico como aprendizaje o enseñanz̧a. La didáctica es suficientemente rica y flexible como para albergar en sus unidades de apropiación (como aprendizaje o enseñanza), distintas improntas, intenciones y propósitos propios. En el proceso de apropiación cada estudiante poblará a estos términos de improntas y sesgos propios al adaptarlas para sí desde el habla del grupo clase.

Rogoff (1995) utiliza el término apropiación en la literatura educativa para enfatizar la naturaleza social y dialógica del proceso de aprendizaje. En el ámbito de la educación científica el término apropiación ha sido también utilizado para denotar un aprendizaje que es portador de construcción de identidad, estableciendo un nexo entre aprendizaje y construcción de 
identidad (De Ambrosis y Levrini 2010). Esta idea es adaptada y enriquecida por Levrini et al. (2015) al posicionar a la apropiación como el nexo entre el aprendizaje disciplinario productivo (Engle y Conant 2002) y la construcción de la identidad (Cobb et al. 2009), poniendo el foco en el cuestionamiento sobre cómo el aprendizaje disciplinario productivo puede ser el lugar en el cual los estudiantes pueden construir una narrativa de sí mismos, es decir, construir identidad. En otras palabras, las autoras se preguntan sobre cómo una disciplina puede generar espacios y actividades no sólo para aprender, sino para desarrollar identidad. La definición tentativa inicial sobre la apropiación que trabajan estas autoras es la siguiente:

"Apropiación es un proceso complejo y reflexivo de transformación del discurso cientifico (palabras y afirmaciones cientificas) que alberga la bistoria personal de uno mismo, pero que al mismo tiempo respeta las reglas y restricciones disciplinarias. El proceso de transformación involucra poblar el discurso científico con las propias intenciones, gustos idiosincráticos y propósitos, a los fines de hacerlo sensible no sólo a uno mismo sino también a la manera de participar en el contexto social de la clase" (Levrini et al. 2015, pp 99).

La fortaleza del trabajo de estas autoras reside en la generación de una definición operacional (indicadores) que transforma la definición anterior en una que permite reconocer cuándo ocurre (o no) el proceso de apropiación en el aula. Según ellas, la apropiación puede ser reconocida cuando el discurso de los estudiantes cumple con los siguientes indicadores:

A- Se desarrolla a partir de un conjunto de palabras o expresiones que se repiten en varias oportunidades y que expresan una idea idiosincrática, auténtica y personal con respecto al contenido de física que están trabajando. La idea es reconocible como auténtica e idiosincrática porque es diferente de las de otros estudiantes y la elección lingüística, tanto como el tono que utiliza, no es "prestado" de la autoridad (del profesor, del texto, de otro compañero autorizado).

B- Es disciplinariamente fundamentado, es decir la idea idiosincrática es usada por el estudiante como una herramienta para seleccionar piezas del conocimiento normativo y para coordinarlas respetando las reglas y principios de la física.

C- Es profundo, es decir, la idea idiosincrática debe involucrar la dimensión metacognitiva (qué significa para mí el aprendizaje de la física) y epistemológica (cuál imagen de la física tiene sentido para mî).

D- Es no incidental, es decir que la idea idiosincrática pueda ser rastreada hacia atrás en distintas situaciones y actividades de clase y, por lo tanto, es reconocible dentro de la historia personal del estudiante.

E- Es portador de relaciones sociales, es decir, que la idea idiosincrática posicione al estudiante dentro de una comunidad de clase y viceversa, que la dinámica de la clase sea inseparable de la idea idiosincrática.

En este trabajo se recuperan estos indicadores, no para referirnos a la apropiación de conceptos físicos, sino para referirnos a la apropiación de la enseñanza y el aprendizaje, en un curso de didáctica de la física para futuros profesores de física. Tomamos a esta definición operacional (indicadores) como referente teórico porque creemos que rescata e ilumina suficientemente bien el tipo de involucramiento personal observado en los estudiantes de profesorado alrededor de sus concepciones de aprendizaje y enseñanza.

Concretamente, las preguntas que guían este estudio son:

¿Cómo es la apropiación de la enseñanza y el aprendizaje de los futuros profesores de física durante el cursado de la asignatura Didáctica de la Física? ¿Cuáles son las ideas auténticas y personales con las que los futuros profesores producen sus discursos en relación a la enseñanza y al aprendizaje? 


\section{Registros y participantes}

La población estudiada son seis estudiantes de un profesorado de física perteneciente a una universidad pública de Argentina que habían cursado y rendido el curso de Didáctica de la Física. Como práctica de evaluación de su tarea, los docentes del curso de Didáctica de la Física, solicitaron a los estudiantes una devolución individual sobre el desarrollo de la asignatura en dos momentos del año: a mediados del año lectivo y al final del mismo, en el momento del examen final. Los estudiantes del curso eran nueve, de los cuales seis rindieron el examen final de la asignatura y presentaron las dos devoluciones antes mencionadas. Los tres estudiantes restantes no terminaron el cursado de la materia en ese año. Estas doce devoluciones, dos por cada uno de los seis estudiantes, fueron seleccionadas como los registros de este estudio. La consigna dada a los estudiantes al final de la asignatura fue la que se muestra en la Figura 2. Una consigna similar fue dada a mediados del desarrollo del curso. La decisión de seleccionar estas devoluciones como registros para esta investigación fue posterior a su diseño y utilización.

A modo de cierre de la materia los invitamos a reflexionar respecto del recorrido que hemos realizamos a lo largo del año. Para ello, les pedimos que realicen por escrito comentarios acerca de aquellas cuestiones que consideren oportunas mencionar. Como docentes del espacio, consideramos de gran relevancia sus aportes por lo que les pedimos que sean los más desarrollados posibles. Dicha devolución puede abordar las dimensiones que prefieran, sin embargo, les dejamos una serie de cuestiones que podrían considerar:

- Aspectos y/o actividades que más les gustaron y las que no

- Percepciones respecto de lo que consideraban que se trabajaría en esta materia: ¿Algo cambió?

- Metodologías de trabajo empleadas; ¿Qué percibieron en una clase en particular y/o unidad?, ¿Cambiaron sus percepciones al inicio y al final?

- Expectativas logradas y no logradas, personales y/o referidas a la materia

- Qué cambiarían; qué no cambiarían; sugerencias para el desarrollo del próximo año en esta materia.

Figura 2. Consignas dadas a los futuros docentes para hacer sus devoluciones sobre la materia.

Estos seis estudiantes tuvieron un buen desempeño durante todo el curso, cumpliendo en tiempo y forma con las actividades propuestas a lo largo de todo el año. La asistencia promedio anual a las clases fue superior al $80 \%$ en todos los casos. Las respuestas y las discusiones originadas a partir de las actividades planteadas al interior de cada bloque, eran utilizadas por los docentes para realizar evaluaciones formativas que permitían hacer un seguimiento de sus procesos de aprendizaje y hacer cambios cuando era necesario. Al finalizar cada bloque, los futuros docentes realizaban una evaluación de acreditación. Las notas de esas evaluaciones (que fueron siete, una para cada bloque) estuvieron siempre comprendidas entre 6 (seis) y 10 (diez). Cada uno de los seis estudiantes finalizó el cursado de la materia con un promedio de sus evaluaciones parciales superior o igual a 8 (ocho). Todos ellos finalizaron en tiempo y forma la unidad didáctica requerida por la cátedra al final de la asignatura. La presentación y defensa de esa unidad didáctica fue el examen final de la materia, que todos aprobaron con notas comprendidas entre 9 (nueve) y 10 (diez). Las devoluciones que los estudiantes realizaron sobre la materia (Figura 2) no formaban parte del seguimiento ni de la acreditación de la misma, sólo fueron instrumentos que el grupo docente utilizó para evaluar la enseñanza. La extensión de las devoluciones osciló entre menos de una página y tres páginas de longitud. Es importante aclarar que estas devoluciones fueron producidas por los estudiantes con anterioridad a la decisión de iniciar el proceso investigativo que aquí se reporta. Por ello no hay (ni podría haber) relación alguna entre las preguntas planteadas en la devolución (Figura 2) y los indicadores de apropiación. Estas devoluciones, que fueron seleccionadas del conjunto de producciones de los estudiantes a posteriori del cursado de la materia, son consideradas como los registros de la investigación actual. 


\section{Aspectos metodológicos: Adaptación de los indicadores de apropiación a los discursos de los futuros docentes de física}

Los indicadores elaborados por Levrini et al. (2015) fueron aplicados a los discursos de los futuros profesores a fin de evaluar su potencia operacional en este nuevo contexto, haciendo visible lo invisible. Los discursos de los futuros docentes (extraídos de las respuestas a la actividad de la Figura 2) eran leídos en sucesivas oportunidades de manera independiente por cada uno de los autores de este trabajo, hasta advertir la emergencia de los cinco indicadores antes descriptos. Luego de las lecturas y análisis individuales, los autores se reunían para discutir sus interpretaciones en relación a cada uno de ellos. Este procedimiento se repetía hasta llegar a un consenso entre ellos (Hammer y Berland 2013, Merriam y Tisdell, 2016, Creswell y Poth 2018).

Lo primero que analizamos en los discursos de cada futuro docente fueron esas ideas/expresiones personales e idiosincráticas para referirse al aprendizaje y a la enseñanza, y que potencialmente se constituyera en el sello personal de ese futuro docente. Analizábamos si esas expresiones se repetían varias veces en sus discursos y cómo se utilizaban en cada ocasión que lo hacían, a fin de validar la coherencia de tal idea o expresión. Ese trabajo fue realizado individualmente por cada autor y mediante lecturas sucesivas de los registros. Luego, los tres autores compartían sus interpretaciones alrededor de este punto y, tras algunas iteraciones, llegaban a un acuerdo en relación a cuáles eran esas expresiones personales e idiosincráticas que constituían el sello personal de cada futuro docente para referirse al aprendizaje y a la enseñanza. Para este análisis, los tres autores llegábamos a un acuerdo luego de dos o tres iteraciones. En algunos casos estas expresiones eran más fáciles de reconocer en los discursos que en otros y, por lo tanto, los tres autores llegábamos más rápidamente a un acuerdo. En cinco de los seis casos fue posible encontrar esta expresión, esta idea personal e idiosincrática que se repetía en más de tres oportunidades en los discursos para referirse al aprendizaje y a la enseñanza. De esta manera trabajamos para lograr consensos en la interpretación de los indicadores A y $\mathrm{D}$ a partir de los discursos de los futuros docentes.

Una vez identificada esa expresión idiosincrática en relación al aprendizaje y a la enseñanza en la historia personal de cada futuro docente, procedíamos a validar si esa idea/expresión personal respetaba las normas de la disciplina. En este caso, la didáctica de las ciencias. Para ello analizábamos si la idea/expresión idiosincrática y personal en relación a la enseñanza y al aprendizaje, podía ser interpretada a través de algún resultado consensuado en el campo de la didáctica de las ciencias (indicador B). Todas las ideas/expresiones fueron identificables con algún resultado en el campo de la didáctica de las ciencias. En todos los casos, se requirieron una o dos reuniones entre los autores para llegar a un consenso.

Luego buscábamos en los registros si la idea personal e idiosincrática era profunda, es decir, si involucraba una dimensión metacognitiva que expresara una postura personal, una reflexión sobre su propio aprendizaje. Esta dimensión metacognitiva era reconocible a través de expresiones como "me di cuenta", "me impresionó", "fue lo más fuerte que nos pasó", "superó mis expectativas", "fui capaz de experimentar", etc. Esta característica, que según la definición operacional elegida coincide con el indicador $\mathrm{C}$, fue fácilmente reconocible en los discursos de los futuros docentes y requirió poca discusión, llegando fácilmente a un consenso entre las interpretaciones individuales de los autores.

Por último, el indicador E, que denota la dimensión social del aprendizaje, las formas en que las ideas personales e idiosincráticas (indicador A) posicionan al futuro docente en el colectivo de la clase, fue el más de simple identificar en los registros. Cuando los futuros docentes se expresaban en relación a sus experiencias durante el curso, la mayor parte de las veces lo 
hacían en primera persona del plural, como si no fuera posible desacoplar estas experiencias de las de sus compañeros. En este sentido, la dimensión colectiva del aprendizaje experimentado fue quizás lo más sobresaliente en los discursos de los estudiantes.

Como se presentará con detalle más adelante, y a partir del proceso interpretativo antes descripto, fue posible determinar la presencia de los cinco indicadores (es decir de reconocer apropiación) en cinco de los seis casos involucrados en el estudio. En aquellos casos en los que se reconoció apropiación no surgieron dudas que nos hicieran pensar que la apropiación había sido parcial. Sin embargo, dado que la apropiación es un proceso complejo y reflexivo de transformación del discurso científico, es posible que no siempre sea el caso poder decidir con relativa claridad si ese proceso fue logrado o no. En este estudio no ha sido necesario definir "grados" de apropiación, aunque no descartamos que fuera necesario en otro contexto y que pudiera ser algo sobre lo que trabajar en el futuro. En nuestros registros encontramos que, una vez identificada la idea idiosincrática y personal, distintiva de la historia personal de cada futuro docente (quizás el indicador que implicó más discusiones entre los autores), los otros se desprendieron bastante naturalmente, como si estuvieran entrelazados con esa idea.

\section{Resultados}

Se organizan los resultados en torno a las ideas idiosincráticas y personales de los estudiantes del curso. Se transcriben extractos del discurso de los estudiantes que permiten reconocer la apropiación a través de los indicadores A, B, C, D y E presentados anteriormente. Aunque estos indicadores se construyeron inicialmente para reconocer la apropiación de conceptos físicos y fueron obtenidos en el contexto de estudiantes secundarios (Levrini et al 2015), aquí se adaptan para reconocer la apropiación de los conceptos de aprendizaje y enseñanza en el contexto de profesores de física en formación. Concretamente, para reconocer apropiación en futuros docentes durante el curso de la asignatura de Didáctica de la Física. Los nombres de los estudiantes son ficticios.

\section{Eloísa: “Aprender (a enseñar) es saber recetas vs. aprender (a enseñar) es un proceso de construcción individual y grupal”}

En la devolución a mitad de año Eloísa expresa que cambió su concepción de aprender y de enseñar desde su idea inicial que era aprender a enseñar es aprender un conjunto de recetas. Según su idea inicial, las recetas son un conocimiento que el docente ya posee y que simplemente imparte a los futuros profesores:

Mi percepción al inicio de la materia fue muy diferente con la que tengo al finalizar la misma. Pensé que las clases serian del tipo autoritativas, con el docente al frente impartiendo saberes, dándonos recetas para la elaboración de actividades... Y mi sorpresa fue grata cuando fui descubriendo que las clases tenian un enfoque totalmente distinto.

Esta visión inicial de Eloísa, con la que llegó al curso de Didáctica de la Física, atribuye al docente el rol de proveer conocimiento, y al estudiante, el rol pasivo de recibirlo. Por lo tanto, según esta idea inicial, enseñar es proveer conocimiento y aprender es recibirlo. Eloísa expresa su sorpresa al descubrir que en el curso el enfoque era totalmente distinto.

En la devolución anual, Eloísa expresa claramente otra concepción de lo que es aprender y enseñar y la compara con su concepción inicial, expresada nuevamente en términos de recetas (indicador D). En el fragmento que sigue, Eloísa plantea una oposición entre aprender a enseñar como aprender recetas, y otra en la que la aprender a enseñar es aprender a propiciar en el aula un clima en el que las ideas son escuchadas y en el cual hay lugar para un proceso de construcción personal y colectivo. Además, se trata de una idea idiosincrática porque es 
personal, producto de su vivencia (a modo personal, durante el transitar... pude vivenciar...). Una idea que claramente no proviene de la autoridad (indicador $A$ ):

Me di cuenta que no existian tales recetas sino que todo es parte de un proceso de construcción personal y grupal. Más aún, el vinculo que se fue propiciando en el aula fue y es muy favorecedor, todos nos sentimos participes, que nuestras ideas se escuchan y son tomadas en cuenta, no es algo vertical. A modo personal, durante el transitar de esta materia pude vivenciar un crecimiento personal, asi como también un crecimiento a nivel académico.

Este fragmento también denota un proceso metacognitivo, en el que ella se da cuenta que no existen tales recetas. Eloísa reflexiona sobre su propio proceso de aprendizaje y advierte el cambio en su concepción de enseñar y de aprender (indicador $C$ ). También se puede advertir que sus nuevas ideas sobre qué es aprender y qué es enseñar están en sintonía con las concepciones contemporáneas constructivistas sobre estos dos conceptos provenientes de la didáctica de las ciencias (indicador B).

Eloísa también hace referencia explícitamente al grupo de compañeros y expresa que aprender es una actividad colectiva que la posiciona intelectualmente (ver otros puntos de vista y poder llegar a un consenso) y emocionalmente en el grupo (más confianza en la manera de dirigirme a mis compañeros y profesores) (indicador E).

Por ello las actividades que más me gustaron fueron las grupales, pues el trabajo en equipo sirvio para fortalecer las aptitudes personales y además a ver otros puntos de vista y poder llegar a un consenso... Pude desenvolverme ante la clase, actividad que siempre me costó, pero que al pasar a hacer las exposiciones orales fui tomando más confianza en la manera de dirigirme a mis compañeros y profesores...

\section{Renata: "La enseñanza centrada en el docente vs. la enseñanza centrada en el estudiante"}

Renata repite una idea en ambas devoluciones que pone en evidencia cuál es el centro de la enseñanza, alrededor de quién ocurre principalmente, sobre quién está puesto el foco. Ella contrapone la idea de enseñanza centrada en el docente con la enseñanza centrada en el estudiante. Esta idea es auténtica y personal, y remite a sus emociones (es lo más fuerte que nos pasó... ya no vemos al proceso de enseñar como...) (indicador $A$ ).

Cambió nuestra visión de enseñar, que es lo más fuerte que nos pasó, ya no vemos al proceso de enseñar como algo centrado en el docente, como un volcado de conocimiento del profesor hacia los alumnos sino, como una construcción del conocimiento de los estudiantes, a partir de trabajar sobre lo que ellos ya traen (ideas previas).

En la devolución al final del año, se encuentra nuevamente esa idea personal e idiosincrática (indicador D) que refiere a la oposición entre centrar la enseñanza en el docente o centrar la enseñanza en el estudiante. En su discurso se advierte una clara componente metacognitiva (me sorprendió... fue dificil cambiar de mentalidad... entender que sin ellos y sin su intervención la clase no tiene sentido) refiriéndose a su propio proceso de cambio, a cómo lo transitó y qué significó para ella (indicador $C$ ).

A mi en lo personal me sorprendió la materia en todo momento, pensé que me enseñarían a dar clases, a enseñar, a llenar el libro de temas. Me gustó muchísimo el enfoque de la materia, rompiendo con la enseñanża a la que estamos acostumbrados y con la que sabemos que no está funcionando. Fue difícil cambiar la mentalidad, fue todo un proceso cambiar el enfoque de dejar de centrar la clase en el docente a pasar a centrar la atención en los estudiantes, a entender que sin ellos y sin su intervención la clase no tiene sentido.

La idea de centrar la clase en el estudiante partiendo desde lo que ya saben es una idea reconocida como fundamental en la didáctica desde hace más de 30 años, de modo que podemos reconocer que la idea de Renata respeta el conocimiento de la didáctica de las ciencias (indicador B). 
Renata en todo momento se posiciona como parte del colectivo de la clase, utilizando la primera persona del plural (nos pasó... no vemos... ya sabemos). Ella también explica cómo el trabajo grupal tuvo un impacto positivo en su experiencia personal, valorando el aporte intelectual de los debates y los consensos (indicador E). Algo que ella no había experimentado previamente.

Creo que esta materia me enseñó a trabajar en grupo, cosa a la que no estaba acostumbrada. Me enseñó a debatir, a tratar de llegar al consenso en las decisiones de los trabajos con mis compañeros... Al principio pensaba que la materia no me iba a gustar, que iba a ser aburrida como pedagogía o psicología, pero la verdad que me encantó. Me encantaba ir a la clase, estar con mis compañeros y con los profes. Me encantó el vínculo que armamos entre todos.

\section{Nacho: "Los contenidos y las actividades de la materia son las herramientas clave para la enseñanza"}

En reiteradas oportunidades Nacho hace referencia a la importancia de los contenidos trabajados en la asignatura y a las actividades realizadas. Él reconoce en esos elementos el mayor aporte a su formación, su principal fuente de aprendizaje. Nacho ya había cursado la materia en otro año, con otro equipo docente, pero no había rendido el examen final. Por ello decidió volver a cursarla en esta oportunidad.

Estoy equilibradamente satisfecho con la forma que se dicta este año. Cada texto tiene su fundamento y su actividad para que podamos por nosotros mismos darnos cuenta de la importancia de los contenidos... siento que Didáctica es una materia elemental en la carrera.

Más adelante Nacho vuelve a mencionar los contenidos y las actividades (indicador $D$ ) de la asignatura como lo más importante para su aprendizaje. La idea de Nacho se centra en las actividades y los contenidos del curso como aquello que le permitió aprender a enseñar. Las palabras contenidos y actividades se encuentran a menudo en su discurso (indicador A), expresando su idea personal sobre el rol que cumplen para aprender a enseñar física. Es una idea personal y auténtica con una fuerte impronta metacognitiva (estamos mejorando, eso es increíble... me gusta el equilibrio... me encanta) (indicador $C$ ).

Me gusta el equilibrio de variedad de actividades que tenemos. Hemos hecho laboratorio, entregas por email, exposiciones, investigaciones y no hay una que se destaque demasiado ante las otras es decir que hay un perfecto equilibrio y nada se hace repetitivo. Los debates de cierre en cada clase siempre son serios, apuntamos a cosas y contenidos concretos y nos vamos con algo concreto a casa, eso me encanta.

En la devolución final, Nacho vuelve a expresar en reiteradas oportunidades el rol fundamental de los contenidos y las actividades realizados durante el curso para su formación.

Ayer mismo pude refrescar cada uno de los contenidos de los bloques que estuvimos trabajando durante el año y con ello hacer una retrospectiva de mi desempeño personal y punto de vista de la materia... Ahora que miro los contenidos de los bloques pude entender mucho mejor la relación entre ellos y la importancia de cada uno... me gustó mucho, en cada clase podíamos entender (gracias a las actividades que haciamos) cómo relacionar los contenidos bibliográficos que leíamos con la puesta en práctica y esta relación de no ser trabajada en la materia para uno mismo resulta de mucha dificultad entenderla, por ejemplo, leer un paper de ideas previas y entender cómo hacer una buena actividad para recabarlas, tienen una relación, pero es complicado desarrollarla solo.

Podemos decir que Nacho, a través de su mirada retrospectiva y metacognitiva sobre la asignatura en relación a los contenidos y las actividades, coincide con un principio básico de la enseñanza en el marco de la didáctica de las ciencias, que es el desarrollo de los contenidos a través de actividades (a menudo referido como aprendizaje por indagación). Si bien Nacho no expresa esta idea con esas palabras, en varias oportunidades él la insinúa: me gustó mucho, en cada clase podiamos entender (gracias a las actividades que haciamos) cómo relacionar los contenidos con... o en 
por ejemplo, leer un paper de ideas previas y entender cómo hacer una buena actividad para recabarlas, tienen una relación, pero es complicado desarrollarla solo. Nacho pone en valor a la actividad como mediadora para la comprensión de los contenidos. De manera que la idea de Nacho respeta una idea consensuada en el campo de la didáctica de las ciencias, como es el aprendizaje por indagación (indicador B).

Nacho hace referencia al trabajo en grupo como una experiencia singular y una instancia para aprender desde los diferentes puntos de vista. Él no solo advierte qué ha aprendido, sino cómo sus compañeros lo han hecho, posicionándose en el grupo clase como un observador singular (indicador E).

Me pareció tremendamente fructifero realizar una exposición grupal de algún trabajo en cada cierre de bloque... Me gustó que hayamos rotado los grupos de trabajo, asi nos enfrentábamos a diferentes puntos de vista o formas de trabajar constantemente... hemos mejorado y yo me doy cuenta, como mis compañeros van mejorando, eso es increíble y es algo que es casi imposible de detectar en otras materias.

\section{Luis: "Sentirse acompañado en el proceso de aprender"}

La idea personal de Luis resalta la importancia del acompañamiento durante el aprendizaje. El seguimiento y el acompañamiento son lo más importante para él. Las palabras continuo seguimiento y acompañado se repiten en su discurso para referirse al proceso de aprendizaje (indicador $A$ ).

Conocía los temas de la materia porque cursé hace dos años gran parte de la materia con otros profes, y me gustó mucho más las metodologias que se emplean ahora, ya que me parece muchisimo mejor que los trabajos se realicen en grupo. Además, me parece muy bueno que baya un continuo seguimiento sobre las elaboraciones de nuestros trabajos, lo que permite avanzary no atascarse con una actividad.

Luis vuelve a referirse al seguimiento y acompañamiento durante su proceso de aprendizaje en la devolución del final de año (indicador D). Él refiere a un ejemplo durante el desarrollo de uno de los contenidos en los que ellos experimentaron una dificultad. Luis rescata el valor del seguimiento continuo como sinónimo de evaluación formativa, como acción indispensable para reorientar el curso de la enseñanza. Esta función de la evaluación formativa, la de evaluar para mejorar la enseñanza, es ampliamente reconocida en el ámbito de la didáctica, por lo que podemos reconocer que la idea de Luis respeta los principios de la didáctica (indicador B).

Con el permanente seguimiento que nos realizaban, teniendo en cuenta también la dimensión bumana de sus clases, me hicieron sentir bastante acompañado en el cursado a lo largo del año. Siempre que estuve y estuvimos trabados con las distintas actividades, ustedes nos brindaban una mano baciéndonos sacar lo mejor de nosotros, evitando frustraciones.... Me pareció muy bueno el ejemplo que nos dieron en el bloque 1 cuando vieron que nos costaba comprender algunas cosas (realizar algunas actividades) ... Pensaron otras actividades para lograr el objetivo. Muy bueno, porque fue un buen ejemplo que marcaba la importancia de las evaluaciones formativas.

Luis tiene una idea bastante clara del acompañamiento en su propio proceso de aprendizaje, que está presente en su discurso cuando refiere al desarrollo de la unidad didáctica en el cierre de la materia. Él remarca cómo el debate y el acompañamiento le permitieron avanzar, mostrando una connotación metacognitiva (empecé a ver con otra mirada... me sirvieron para seguir aprendiendo) en relación a su idea personal (indicador $C$ ).

La actividad que más me gustó fue la elaboración de la unidad didáctica; al comienzo sentía que no me gustaba, porque parecian actividades inconexas, pero luego de que llegamos al último modelo de mapa conceptual, empecé a ver con otra mirada al trabajo, ya tenía sentido el orden de los temas por la manera de conectarse uno con el otro y teniendo en cuenta siempre el diseño curricular. Tuvimos buenos debates en la 
elaboración de esta, que me sirvieron para seguir aprendiendo y puliendo cosas. Recibimos muchísimo acompañamiento de los profes, que también nos sirvieron mucho.

Luis en numerosas oportunidades expresa sus experiencias en primera persona del plural, refiriéndose a él pero como parte del grupo de la clase. También se refiere al trabajo grupal como una actividad intelectualmente beneficiosa, aludiendo a cómo se multiplican las oportunidades para aprender en esos espacios. Valora la variabilidad de puntos de vista y cómo eso los obliga a argumentar a favor o en contra de ellos. En síntesis, percibe y valora al aprendizaje como una actividad colectiva, distinguiendo el rol de los individuos y sus ideas en ellos (indicador $E$ ).

Me pareció bastante beneficioso el hecho de que la mayoría de las actividades sean en forma grupal porque permite intercambiar ideas (a veces opuestas), defender nuestras posturas y concluir con producciones mucho más sólidas... mil gracias a mis compañeros, estoy muy contento con todos los resultados en el grupo a lo largo del año. Dana: "Los contenidos y las actividades de la materia son herramientas clave para
planificar la enseñanza en el futuro y también para aprender mejor la física"

Las palabras que se repiten varias veces en el discurso de Dana son contenidos, actividades, herramientas para el futuro y aprender física. Similar a la idea idiosincrática de Nacho, pero con otra impronta, Dana percibe que su aprendizaje sobre la enseñanza está muy fuertemente influenciado por los contenidos trabajados y por las actividades realizadas en las clases. Ella denota, también en varias oportunidades, que ese aprendizaje será clave para desenvolverse como futura docente en la planificación de sus clases.

Voy a comenzar diciendo que la materia ha superado mis expectativas en cuanto al desarrollo de los contenidos puedo decir que la metodología del desarrollo de las clases, las actividades, el seguimiento y las evaluaciones han sido diferentes, interesantes y muy productivas para adquirir un sin fin de herramientas... estoy convencida me servirán para mi futuro desempeño como profesora de ciencias, en este caso en el área de la física... los textos presentados para lectura e interpretación me han resultado además de interesantes, de fácil comprensión y análisis, aportando muchos contenidos... la forma de evaluación, distinta para cada bloque, fue novedosa e interactiva, no remitiéndose solo a la evaluación convencional... Quiero ser una profesional comprometida con un aprendizaje significativo y provechoso en todas las dimensiones. Sé que todo lo trabajado y adquirido en esta materia será sumamente importante a la hora de planificar clases y/o actividades... Haciendo un balance, desde principio de año hasta abora, me doy cuenta de todo lo que hemos logrado, el trabajo en grupo, que como siempre menciono me ha aportado muchas herramientas nuevas y significativas.

Diversas expresiones muestran la dimensión metacognitiva de la idea de Dana en relación a su aprendizaje durante el curso: ha superado mis expectativas... estoy convencida... sé que todo lo trabajado y adquirido en esta materia será sumamente importante... haciendo un balance me doy cuenta de todo lo que hemos logrado (indicador $C$ ).

Otra impronta personal de Dana es su percepción de que la asignatura le permitió, además de aprender sobre la enseñanza, aprender más de física. Dana expresa que durante el cursado de la asignatura Didáctica de la Física, a través de las situaciones problemáticas de física planteadas, y los debates alrededor de ellas, le permitieron aprender más de física.

Las actividades en que teníamos que resolver problemas de fisica y debatirlos en la clase a veces eran complicadas al principio, pero nos obligaban a pensar mucho... pero el resultado era siempre muy bueno porque aprendiamos física.

En la devolución final, Dana recurre nuevamente a las mismas palabras (indicador D) para expresar cómo ella ha transitado su aprendizaje sobre la enseñanza durante el cursado de la asignatura, mostrando que su idea es idiosincrática y personal (indicador $A$ ). 
El desarrollo conceptual y todas las actividades trabajadas en cada uno de los bloques son piezas fundamentales que seguramente tendré siempre presente a la bora de proyectar todas las actividades que se presenten a futuro. Hemos adquirido también un mejor vocabulario, aprendimos a hacer análisis más críticos y todo eso se ha evidenciado con el avance de las clases... La manera de desarrollar y trabajar cada uno de los contenidos de los bloques, las formas de presentarlos, el modo en que hemos sido evaluados, sus propuestas y todo lo que hemos logrado en la elaboración de la unidad didáctica, son herramientas e instrumentos que nos ayudarán en lo que nos queda de carrera.

También aparece nuevamente la referencia al aprendizaje de la física a partir de la didáctica de la física, que ella luego denomina mirar la física con los ojos de la didáctica.

Les aseguro que todo ha sido de gran utilidad e importancia para nosotros; tanto para esta materia como para poder hacer interrelación con otras. Siento que conceptualmente comprendo mejor la física y sobre todo el objetivo y el propósito de enseñarla... en este proceso de formación docente del que como estudiantes somos partícipes y después de nuestro paso por este espacio curricular, todos ahora podemos ver a la física un poco mejor con los ojos de la didáctica.

Como en el caso de Nacho, la idea idiosincrática de Dana es que los contenidos han sido aprendidos a través de las actividades realizadas (el desarrollo conceptual y todas las actividades trabajadas en cada uno de los bloques... la manera de desarrollar y trabajar cada uno de los contenidos) está en sintonía con las visiones contemporáneas del aprendizaje por indagación, por lo que podemos inferir que la idea de Dana respeta los principios de la didáctica de las ciencias (indicador B). Otra evidencia del mismo indicador puede reconocerse en la retroalimentación que Dana percibe entre la didáctica de la física y la física. Esta relación es reconocida y ha sido objeto de investigaciones en didáctica de las ciencias (Mantyla y Nousiainen 2013, SperandeoMineo et al. 2005), sugiriendo que la retroalimentación entre esos dos campos es recíproca y fructífera para el desarrollo del CDC del profesor.

Para Dana aprender es una actividad colectiva que la posiciona intelectualmente y emocionalmente en relación al grupo (indicador E). Por un lado, casi toda su devolución la realiza en primera persona del plural, denotando su grado de pertenencia al grupo clase. Además, ella se explaya sobre el aporte cognitivo y emocional en relación al trabajo colectivo.

Hemos ido creando un vínculo de confianza tan importante en el aula... Además, espero seguir realizando trabajos en grupos, por lo productivo y las producciones compartidas que se generan... Sin lugar a dudas cursarla ba significado un desafio y un esfuerzo enorme tanto en lo personal como en lo grupal, y sin querer acudir a lo emocional, no puedo dejar de mencionar la emoción y la alegría que me genera ya estar a punto de finalizar con la presentación de la Unidad Didáctica, que hemos diseñado y elaborado con mis compañeros... Estoy convencida de que el cursado de esta materia, las buenas relaciones y vinculos creados entre mis compañeros y también con los docentes de este espacio, han sido para mi un envión muy importante para no volver a dejarme vencer por mis inseguridades y miedos y para continuar motivada con mi carrera.

\section{Fran}

Las devoluciones de Fran fueron muy sintéticas y no se pudieron detectar palabras ni oraciones auténticas y personales en su discurso. Las expresiones de Fran son del tipo: Las actividades que más me gustaron... también me gustó la actividad... se me hizo pesado el bloque 3 con muchas lecturas desde el principio. No se reconocen en su discurso expresiones que permitan reconocer apropiación en relación a la enseñanza y/o al aprendizaje. Fran es tímido y callado, pero fue un buen estudiante a lo largo de todo el curso, con un buen desempeño (y buenas calificaciones) en todas las actividades realizadas. 


\section{Discusión}

En primer lugar, se puede notar que los indicadores para reconocer apropiación, desarrollados por Levrini et al. (2015) en el contexto de estudiantes secundarios aprendiendo física, se adaptan perfectamente bien al contexto de estudiantes de profesorado aprendiendo didáctica de la física. Los resultados muestran cómo esos indicadores pudieron ser fácilmente aplicados en el discurso de los futuros docentes de física para reconocer apropiación en relación al aprendizaje y la enseñanza. Por lo que consideramos que esta extensión de una propuesta teórica/metodológica desarrollada en el contexto de estudiantes secundarios aprendiendo física al contexto de futuros docentes aprendiendo didáctica de la física, ya es un aporte a la investigación en formación docente. En este sentido, el indicador B del contexto original, que hace referencia a que el discurso debe estar disciplinariamente fundamentado, es decir, que debe respetar el conocimiento disciplinario de la física, se pudo adaptar bien al contexto actual. En este trabajo, la referencia disciplinaria no es la física sino la didáctica de la física o de las ciencias, que tiene características epistemológicas propias, distintas de la física. Las ideas idiosincráticas y personales de cada uno de los estudiantes se pudieron identificar con conocimiento producido en el campo de la didáctica de las ciencias de manera clara.

En relación a la primera pregunta sobre cómo es la apropiación de estos futuros docentes, podemos decir que ha sido una apropiación genuina y espontánea ocurrida durante el año transcurrido en Didáctica de la Física. Lo que han aprendido sobre la enseñanza y el aprendizaje (con sus diferentes improntas personales) no ha sido a partir de leer textos o a partir del discurso del profesor, sino a partir de sus experiencias personales sobre aprender y enseñar durante el curso. Ellos mismos experimentaron de manera personal y colectiva, una forma de aprender y una forma de enseñar profundamente distante de sus experiencias previas como estudiantes, que dio lugar a este aprendizaje tan especial, que en este trabajo encuadramos como apropiación. Una característica común a todos los casos estudiados ha sido la valoración del trabajo colectivo, indicando que ahora conciben al aprendizaje y a la enseñanza como fenómenos colectivos. Una idea que tiene mucha aceptación en el campo de la didáctica de las ciencias contemporánea, pero que rara vez se encuentra en las aulas de ciencias.

En relación a la segunda pregunta sobre cuáles son las ideas personales y auténticas relevadas en estos futuros estudiantes, podemos decir que cada una de ellas se corresponde con algún resultado consensuado en el campo de la didáctica de las ciencias. La idea de Eloísa en relación a aprender a enseñar muestra una coincidencia clara con las propuestas socioconstructivistas del aprendizaje, mencionando que éste es un proceso de construcción individual y colectivo. La idea de Renata marca otro principio didáctico también muy consensuado, sobre el lugar que ocupa el estudiante y el profesor en el proceso de enseñanza y de aprendizaje: la enseñanza debe estar centrada en el estudiante y desde lo que éste ya sabe. Renata lleva esta idea hasta el límite, expresando que, sin las participaciones de los estudiantes, sin sus ideas puestas allí, la clase no tiene sentido. Las ideas de Nacho y Dana explicitan el lugar de las actividades en la comprensión de los contenidos, y cómo las primeras cumplen un rol protagónico para el aprendizaje de los segundos. Algo que se asocia a lo que la didáctica de las ciencias denomina aprendizaje por indagación. Dana también hace referencia a otro aspecto interesante en relación al conocimiento didáctico y el conocimiento disciplinario, expresando que la didáctica de la física le ayudó a comprender mejor la física. Esta idea es una de debate actual en la formación docente, que intenta iluminar mejor la intersección y retroalimentación entre el conocimiento pedagógico y el conocimiento disciplinar del profesor. La idea de Luis destaca la función de la evaluación continua en el proceso de aprendizaje, lo que en el lenguaje de la didáctica entendemos cómo evaluar para mejorar la 
enseñanza. Todas estas ideas, que forman parte del núcleo de la didáctica de las ciencias, fueron concebidas por ellos a través de sus experiencias como estudiantes en esta asignatura. El discurso de los estudiantes denota una apropiación personal y auténtica de las mismas. Creemos que este es un aporte relevante para el campo de la formación docente inicial, dado que numerosas investigaciones muestran lo difícil que es para los futuros docentes apropiarse de estas ideas, que están usualmente a contramano de las que traen inicialmente a partir de sus experiencias personales como estudiantes. Más aún, estas visiones a veces persisten durante el ejercicio de la profesión (Guisasola et al. 2013, Melo Niño et al. 2016).

Una implicación para la enseñanza que se desprende de estos resultados es que más que decir lo que hay que hacer para planificar y ejecutar una buena enseñanza, es más productivo hacer lo que decimos a través de planificar y ejecutar una buena enseñanza en los cursos de formación docente. Entendiendo como buena enseñanza aquella dirigida por los resultados contemporáneos de la didáctica de las ciencias. En este sentido, creemos también que este tipo de enseñanza no debería ocurrir únicamente en los cursos de las didácticas específicas sino también en (al menos algunos de) los cursos disciplinares. Esto último genera nuevos interrogantes para los autores de este trabajo que serán objeto de investigaciones futuras.

\section{Conclusiones}

A modo de conclusiones, un aporte distintivo de este trabajo es la validación de una definición operativa de apropiación, construida en otro contexto, para el contexto de la formación de profesores de física. Esta validación permite hacer visible lo invisible. En este caso, hace visible el involucramiento personal de los futuros docentes durante el curso de Didáctica de la Física, en relación a qué es aprender y qué es enseñar, dos conceptos fundamentales en la formación del profesorado.

Como resultado inmediato de la validación anterior, se visibilizó cómo, en un contexto de enseñanza dado, la mayoría de los estudiantes construyeron identidad a través del aprendizaje de la didáctica de la física. Esto aporta un resultado diferente a numerosas investigaciones que reportan lo contrario (Guisasola et al. 2013, Melo Niño et al. 2016). Más aún, la apropiación reportada permite advertir que los modos de apropiación son personales, respetando los gustos e idiosincrasias de cada uno de los futuros docentes. Pero a la vez, permite visibilizar que más allá de esa diversidad, las ideas personales que caracterizan la apropiación están alineadas con la didáctica de la ciencia contemporánea. Esto arroja luz sobre cómo puede ocurrir el involucramiento personal de los futuros docentes en un campo normativo, en este caso, la didáctica de la ciencia.

\section{Referencias}

Acevedo J. A. (2009) Conocimiento Didáctico del Contenido para la Enseñanza de la Naturaleza de la Ciencia (I): El Marco Teórico. Revista Eureka sobre Enseñanza y Divulgación de las Ciencias 6 (1), 21-46.

Bakhtin M. (1981) Discourse in the novel. En M. Holquist (Ed.) The dialogic imagination (pp. 259-422). Austin: University of Texas Press.

Brown B. A. (2006) 'It isn't no slang that can be said about this stuff': Language, identity, and appropriating science discourse. Journal of Research in Science Teaching, 43, 96-126.

Carretero M., (1997) Construiry Enseñar: las Ciencias Experimentales. Buenos Aires. Ed. Aique. 
Cobb P., Gresalfi M., Hodge L. L. (2009). An interpretive scheme for analyzing the identities that students develop in mathematics classrooms. Journal for Research in Mathematics Education 40 (1), 40-68.

Creswell J., Porh C. (2018) Qualiative Inquiry and Research Design. London: SAGE Publications.

De Ambrosis A., Levrini, O. (2010) How physics teachers approach innovation: An empirical study for reconstructing the appropiation path in the case of special relativity. Physical Review Special Topics- Physics Education Research 6, 020107-1-020107-11.

Engle R., Conant F. (2002) Guiding Principles for Fostering Productive Disciplinary Engagement: Explaining an Emergent Argument in a Community of Learners Classroom. Cognition and Instruction 20 (4), 399-483.

Guisasola J., Barraqués J., Garmendia M. (2013) El Máster de Formación Inicial del Profesorado de Secundaria y el conocimiento práctico profesional del futuro profesorado de Ciencias Experimentales, Matemáticas y Tecnología. Revista Eureka sobre Enseñanza y Divulgación de las Ciencias 10 (Núm Extraordinario), 568-581.

Hammer D., Berland L. (2013) Confusing Claims for Data: A Critique of Common Practices for Presenting Qualitative Research on Learning. Journal of the Learning Sciences, DOI: 10.1080/10508406.2013.802652

Levrini O., Fantini P., Tasquier G., Pecori B., Levin M. (2015) Defining and Operationalizing Appropriation for Science Learning. Journal of the Learning Sciences (24) 1, 93-136.

Magnusson S., Krajcik J., Borko H. (1999) Nature, Sources, and Development of Pedagogical Content Knowledge for Science Teaching. En Gess-Newsome, J. y Lederman, N (Eds). Examining Pedagogical Content Knowledge. The Construct and its Implications for Science Education. (pp. 95-132) Dordrecht, Boston, London: Kluwer Academic Publisher.

Mantyla T., Nousiainen M. (2013) Consolidating Pre-service Physics Teachers' Subject Matter Knowledge Using Didactical Reconstructions. Science \& Education DOI 10.1007/s11191-013-9657-7.

Melo Niño L.V., Cañada Cañada F., Mellado V., Buitrago A. (2016) Desarrollo del Conocimiento Didáctico del Contenido en el caso de la enseñanza de la Carga Eléctrica en Bachillerato desde la práctica de aula. Revista Eureka sobre Enseñanza y Divulgación de las Ciencias 13 (2), 459-475.

Mellado V., Borrachero A.B, Brígido M., Melo L.V., Dávila M.A, Cañada F., Conde M.C., Costillo E., Cubero J., Esteban R., Martínez G., Ruiz C., Sánchez J., Garritz A., Mellado L., Vázquez-Bernal B., Jiménez R., Bermejo M.L. (2014) Las emociones en la enseñanza de las ciencias. Enseñanza de las Ciencias 32 (3), 11-36.

Merriam S. y Tisdell E. (2016) Qualitative Research: A Guide to Design and Implementation. New Jersey: Wiley.

Nieva C., Buteler, L., Velasco, J. (2019) Hacer lo que decimos versus decir lo que hay que hacer: Una reformulación en la Didáctica de la Física. Revista de Enseñanza de la Física 31 (Núm Extraordinario), 561-568.

Rogoff B. (1995) Observing sociocultural activity on three planes: Participatory appropriation, guided participation and appendticeship. En J. V. Werstch, P. del Rio, A. Alvarez (Eds), Sociocultural studies of mind (pp. 139-164). Cambridge, England: Cambridge University Press. 
Sperandeo-Minero R., Fazio C., Tarantino G. (2006) Pedagogical Content Knowledge Development and Pre-Service Physics Teacher Education: A Case Study. Research in Science Education 36 (3), 235-268.

Polman J. L. (2006) Mastery and appropriation as means to understand the interplay of history learning and identity trajectories. Journal of the Learning Sciences, 15, 221-259.

Tabak I., Baumgartner E. (2004) The teacher as partner: Exploring participant structures, symmetry and identity work in scaffolding. Cognition and Instruction, 22, 393-429. 